Classification

Physics Abstracts

68.55Ce-61.61.Di

\title{
Time resolved observation of growth processes on Si-surfaces with STM
}

\author{
Ulrich Köhler \\ Institut für Experimentalphysik, Christian-Albrechts-Universität Olshausenstr. 40, D-24098 Kiel, \\ Germany
}

(Received July 4; accepted November 9, 1994)

\begin{abstract}
Time resolved, high temperature STM has been used to study basic steps in homoepitaxial growth of silicon on $\mathrm{Si}(111)$ using disilane as precursor gas. Island nucleation, island growth, step flow and island coarsening after the flux has stopped can directly be imaged on an atomic scale. The shape of island during growth and in thermodynamic equilibrium can be determined. On the $\mathrm{Si}(111)$-surface growth and decay processes are often found to proceed in portions of the surface reconstruction unit cells.
\end{abstract}

The perfect epitaxy of thin films is a key process in modern microelectronics technology. STM has been used extensively in the past ten years to study growth phenomena on a variety of substrates including metal single crystal surfaces [1] and semiconductor surfaces [2].

In the very most cases, STM has been as an ex-situ method in the sense that the structure of the surface was imaged after the growth process, which itself usually involves the substrate being at elevated temperature. On the other hand, growth is a dynamic process quite often far from thermodynamic equilibrium. If the flux is stopped in many cases immediately the structure of the layer changes due to recovering processes. Much effort has therefore been made in assuring that the structure imaged after growth is representative for the structure during growth [3].

In the last three years several studies have been published showing the structure of semiconductor surfaces at elevated temperature [4]. On the other hand, it has also been shown [5, 6] that changes on surfaces induced by adsorption processes can be imaged in-situ with STM. Gaseous species, usually having a sticking probability lower than unity can reach the region directly underneath the tip via multiple collisions.

In this paper we like to combine these two capabilities to study CVD-growth of silicon on a $\mathrm{Si}(111)$-surface by thermal decomposition of disilane $\left(\mathrm{Si}_{2} \mathrm{H}_{6}\right)$ as precursor gas.

The relatively simple case of homoepitaxial growth is governed by only a few elementary steps taking place on an atomic scale [7] (see Fig. 1). Species (in our case Si-atoms and Si-H-species) deposited onto the substrate (A) may diffuse on the surface if the temperature is sufficiently high. 


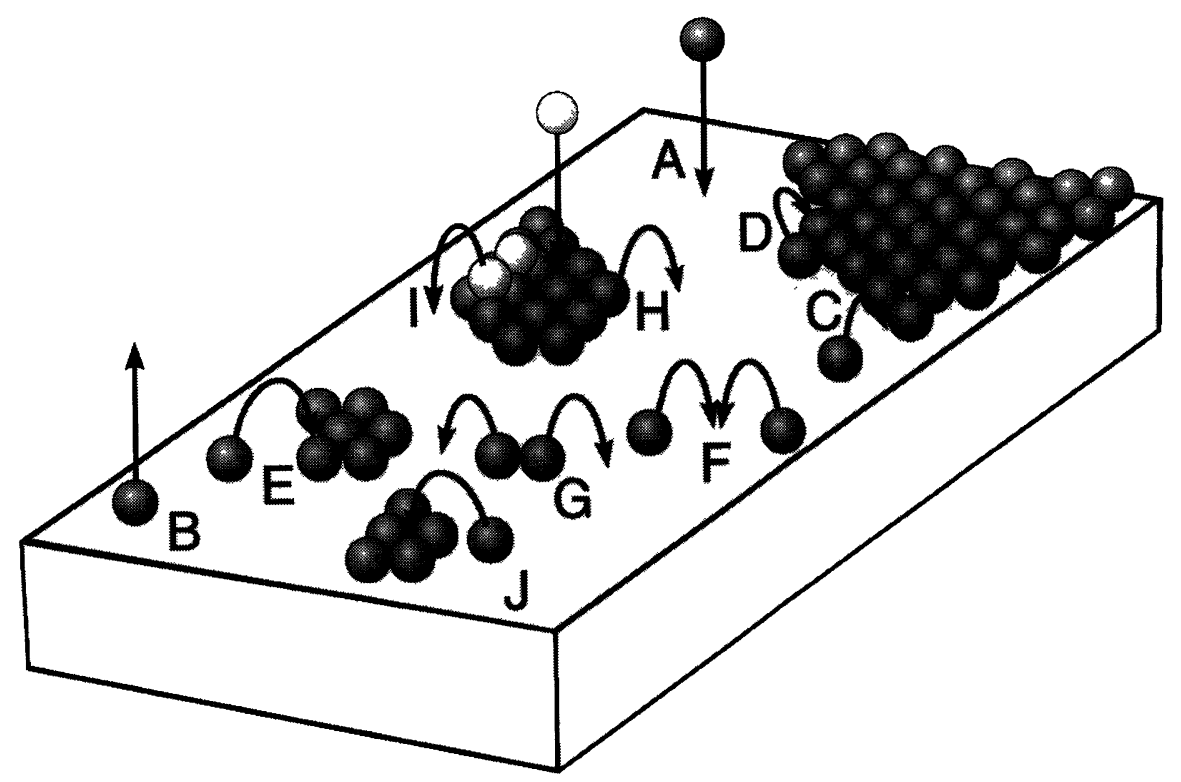

Fig. 1. - Basic steps in epitaxial growth. A) deposition, B) desorption, C) attachment to step edges, D) diffusion along step edges, E) attachment to an island nucleated in an earlier stage of growth, F) formation of a new nucleus, $\mathrm{G}$ ) decay of an understable nucleus, $\mathrm{H}$ ) detachment from a island (basic step in island coarsening), I) flow of atoms across a step edge to a lower level, J) flow of atoms across a step edge to a lower level.

At very high substrate temperature, there is certain probability for the mobile species to desorb again (B) before being accommodated on the surface. The mobile species may reach an already existing step edge (C). Via edge diffusion (D) the shape of the step edge may rearrange. On the other hand, the diffusing species may also attach to an island nucleated in an earlier stage of the growth (E). If the density of mobile species on the substrate increases, two or more of them may meet to form a new nucleus (F). Below a critical size such a nucleus may be unstable and will decay before further atoms can attach (G). Also from bigger islands fragments may detach again $(\mathrm{H})$. In this way the whole structure of the epitaxial layers can rearrange after the deposition has stopped. Larger islands and step edges will grow at the expense of smaller ones to minimize the total length of step edges on the surface.

For technological purposes it quite often is desirable to grow smooth layers. Therefore it is necessary that species landing on top of islands can cross the edge of the island to attach to lower levels (I). An energetic barrier (Schoebel-barrier) may inhibit this down-flow. Of course, also the reverse process may happen, in which a species moves across a step edge from a lower to a higher level (J).

In the following it will be shown which of these basic steps in epitaxial growth can directly be observed using STM.

The experiments were performed in a UHV-chamber allowing a base pressure $<1 \times 10^{-10} \mathrm{mbar}$ necessary for preparation of clean silicon surfaces. During the in-situ growth experiments at elevated temperature the Si-samples were heated by direct DC-current flow. The sample was dosed with the precursor gas using a doser tube directly pointing to the sample in the STM-position. This results in an increased pressure only at the sample and partially compensates the shadowing of the substrate area directly underneath the tip. The unavoidable thermal drift in the microscope at 



Fig. 2. - Nucleation and growth of islands $\left(485^{\circ} \mathrm{C}, p_{\text {disilane }}=1 \times 10^{-6} \mathrm{mbar}\right)$; scan area: $2000 \AA \times 1150 \AA$, total time for whole sequence: $8500 \mathrm{~s}$.

elevated sample temperature is on-line corrected during the scan process. Sequences of typically 20 - 200 images of a specific location on the surface were taken during growth at a temperature as indicated in the figures. Single frames are acquired in $9-85 \mathrm{~s}$, depending on size and speed of 

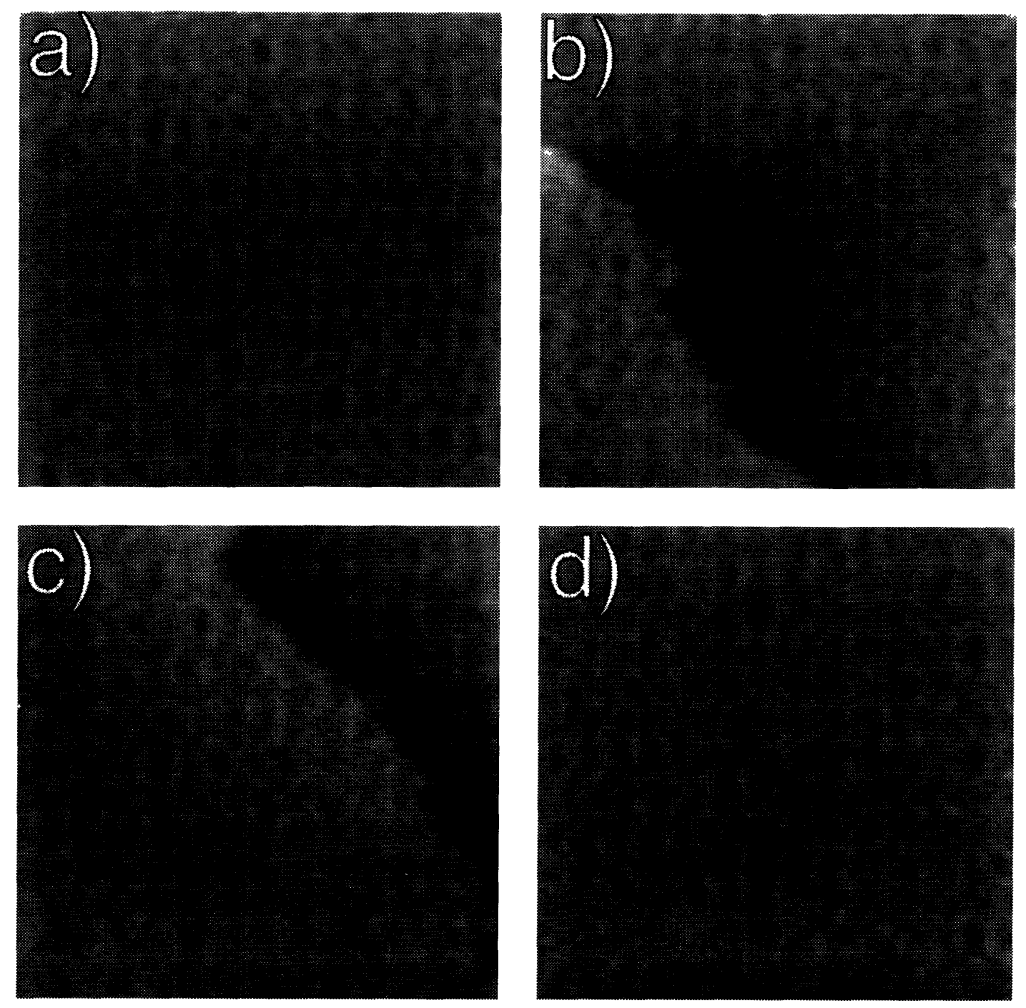

Fig. 3. - Step flow $\left(530^{\circ} \mathrm{C}, p_{\text {disilane }}=4 \times 10^{-6}\right.$ mbar $), \Delta t=20 \mathrm{~s}$; scan area $140 \AA \times 140 \AA$.

the changes on the surface. The figures presented in this paper show selected frames out of these sequences. If the tip-sample bias voltage is kept below $3 \mathrm{~V}$ (positive on negative) no influence of the tip on the growth process was found.

At temperatures around $500{ }^{\circ} \mathrm{C}$ used in the experiments no re-evaporation of silicon into the gas phase (process B in Fig. 1) is expected. Therefore all rearrangement in the stucture of the layer only occurs on the surface.

Figure 2 shows nucleation of triangular islands at $485^{\circ} \mathrm{C}$ (Corresponding to process $\mathrm{F}$ in Fig. 1). Individual diffusing species are not observed because at this temperature they move on a time scale not accessible by STM. The only indication is an increasing noise in the tunneling current with increasing speed of growth. Islands are found randomly distributed over the terrace as well as at the step edge (Fig. 2b upper right corner) displaying homogeneous and inhomogeneous nucleation, respectively. In the further growth these islands grow laterally and vertically (Fig. $2 c, 2 d)$. In the special case of silicon growth on the Si(111) surface it is difficult to grow on the substrate because the perfect termination by the $(7 \times 7)$ reconstruction forms an energetic barrier [8] for the lateral expansion of the islands. Therefore growth is preferred in upper levels leading to the formation of islands up to 6 levels high long before the substrate is covered. A quantitative analysis shows that in this early stage of growth there is a net flux of atoms from the first layer to higher levels (process J in Fig. 1). Nevertheless, the slow lateral growth leads to a coalescence of islands (Fig. 2e, 2f). If one of two coalescing islands is higher than the other immediately the upper level spreads to fill both islands (Fig. 2e, 2f; middle) trying to keep the growth front locally as smooth as possible. 


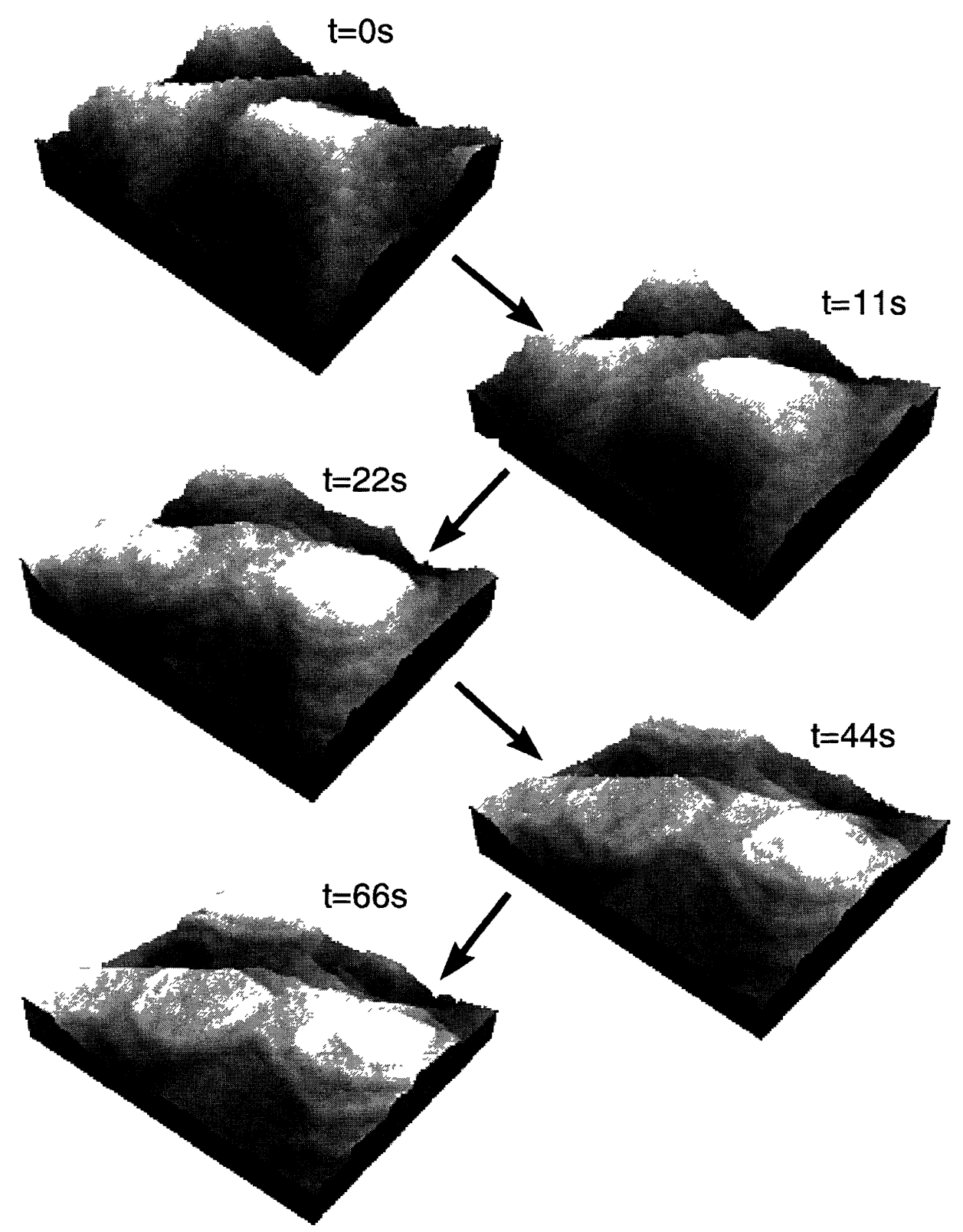

Fig. 4. - Flow across step edge after the flux of disilane has stopped $\left(510^{\circ} \mathrm{C}, 480 \AA \times 550 \AA\right)$. The stage of the upper left frame was caused by a very high flux resulting in a rough surface with a corrugation up to 10 levels high.

On top of such an island growth proceeds by step flow (Fig. 3, process $\mathrm{C}$ in Fig. 1). In the sequence in figure 3 the scale is small enough to resolve individual atoms. In figures $3 b$ and $3 c$ we see a step edge flowing through our field of view. The atomic structure of the growing step edge is visible; it consists of small fluctuation patches of $(7 \times 7)$ and $(5 \times 5)$ reconstructed areas mixed 


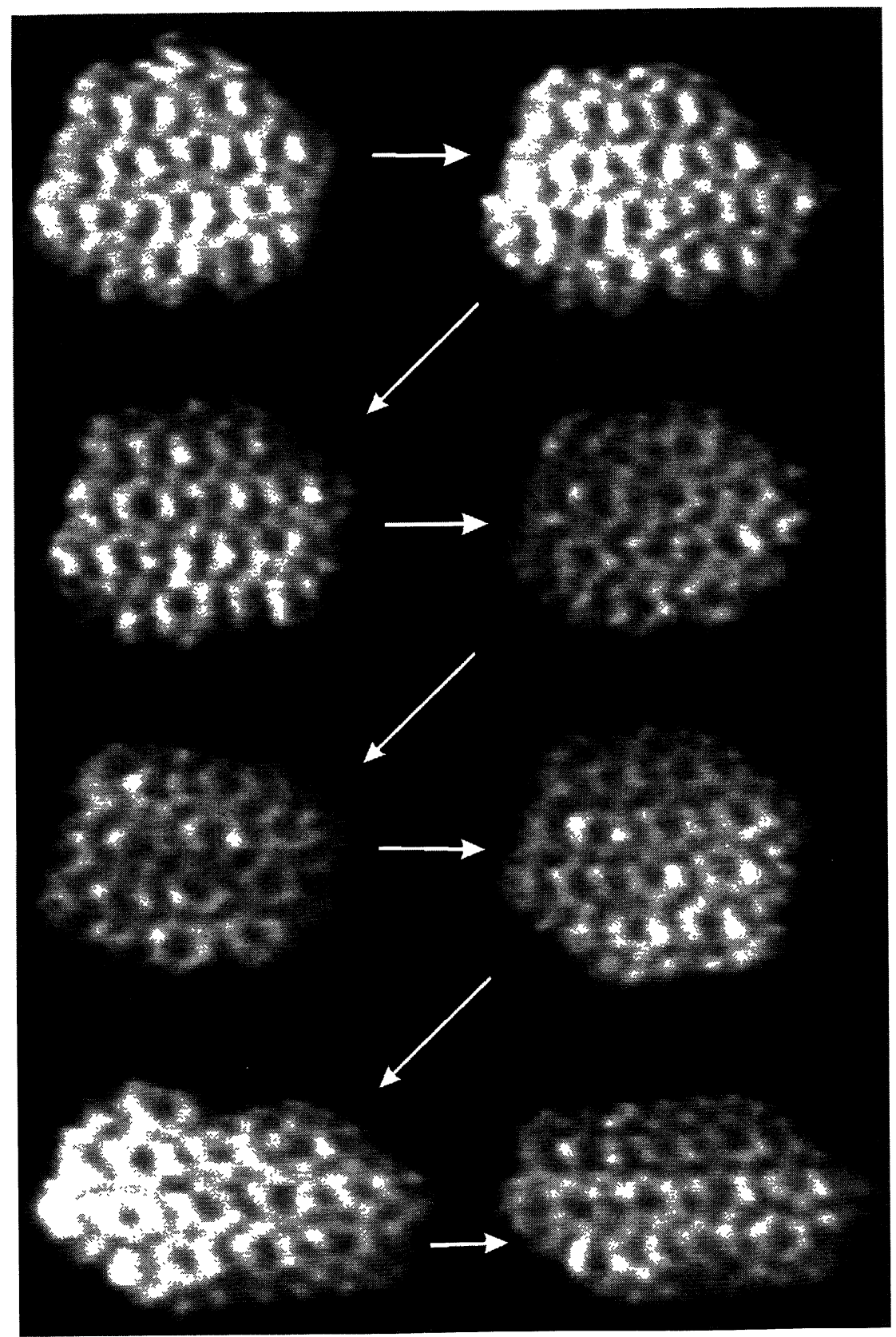

Fig. 5. - Island shape fluctuations at $530^{\circ} \mathrm{C}$ close to thermodynamic equilibrium. Images are shown in steps of $120 \mathrm{~s}$. The arrangement on top of the island shows a $(5 \times 5)$-reconstruction. (Scan area $160 \AA$ $\times 120 \AA)$. 

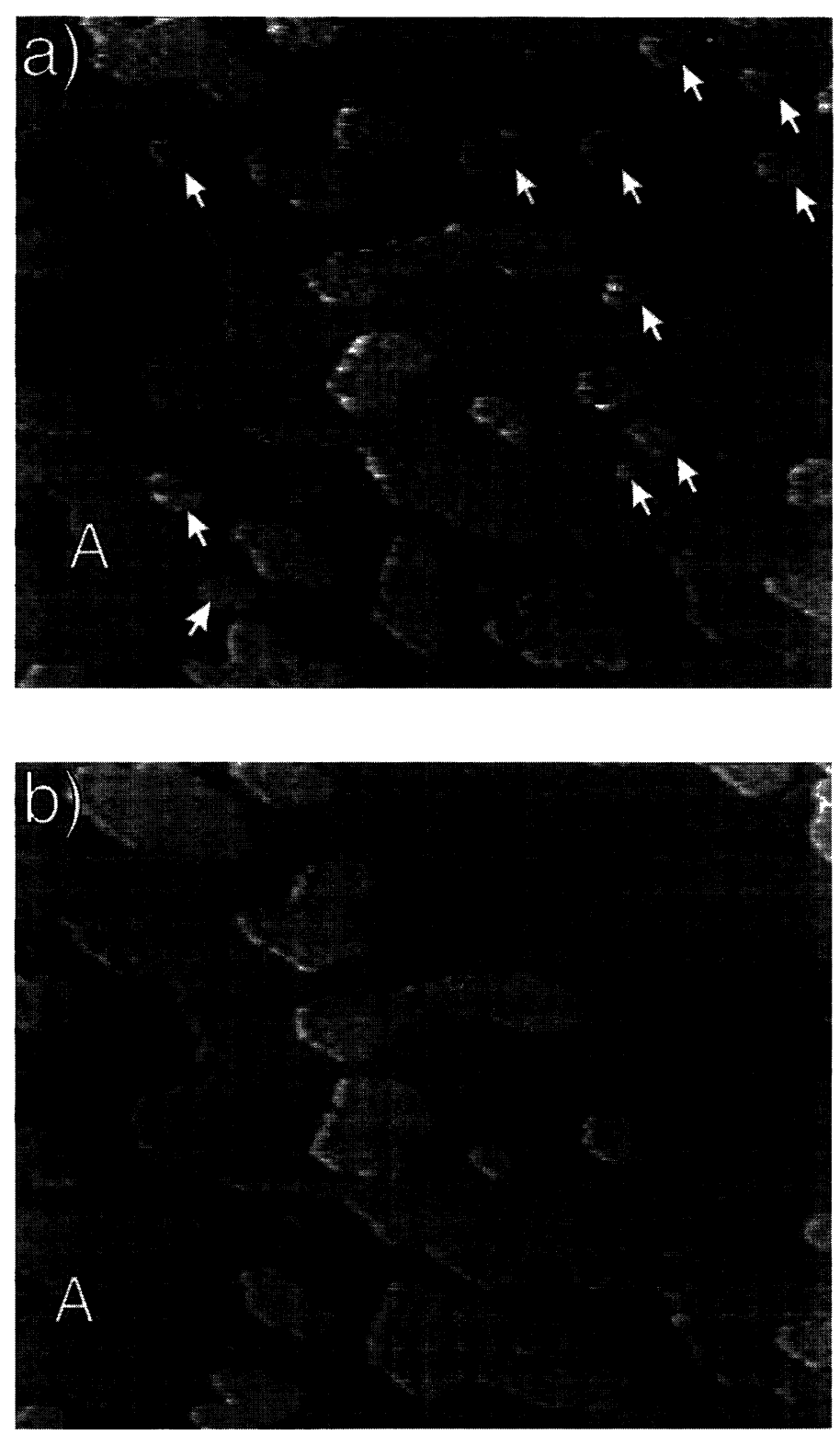

Fig. 6. - Island coarsening at $520^{\circ} \mathrm{C}$. a) shows the state of the surface during growth, b) $1400 \mathrm{~s}$ after the flux has stopped. The arrows in a) point to islands which have disappeared in b). (A) marks a location where a hole in a lower level fills during the coarsening process.

with disordered looking regions. Only after the flux has stopped the surface slowly converts to a perfectly ordered atomic arrangement.

When the arrival rate of disilane molecules is very high, the flux across a step edge is not sufficient to allow layer-by layer growth. Instead, pyramidal shaped stuctures, in the present case up to ten levels high, appear (Fig. $4, t=0 \mathrm{~s}$ ). After the flux has stopped these pyramids decay 

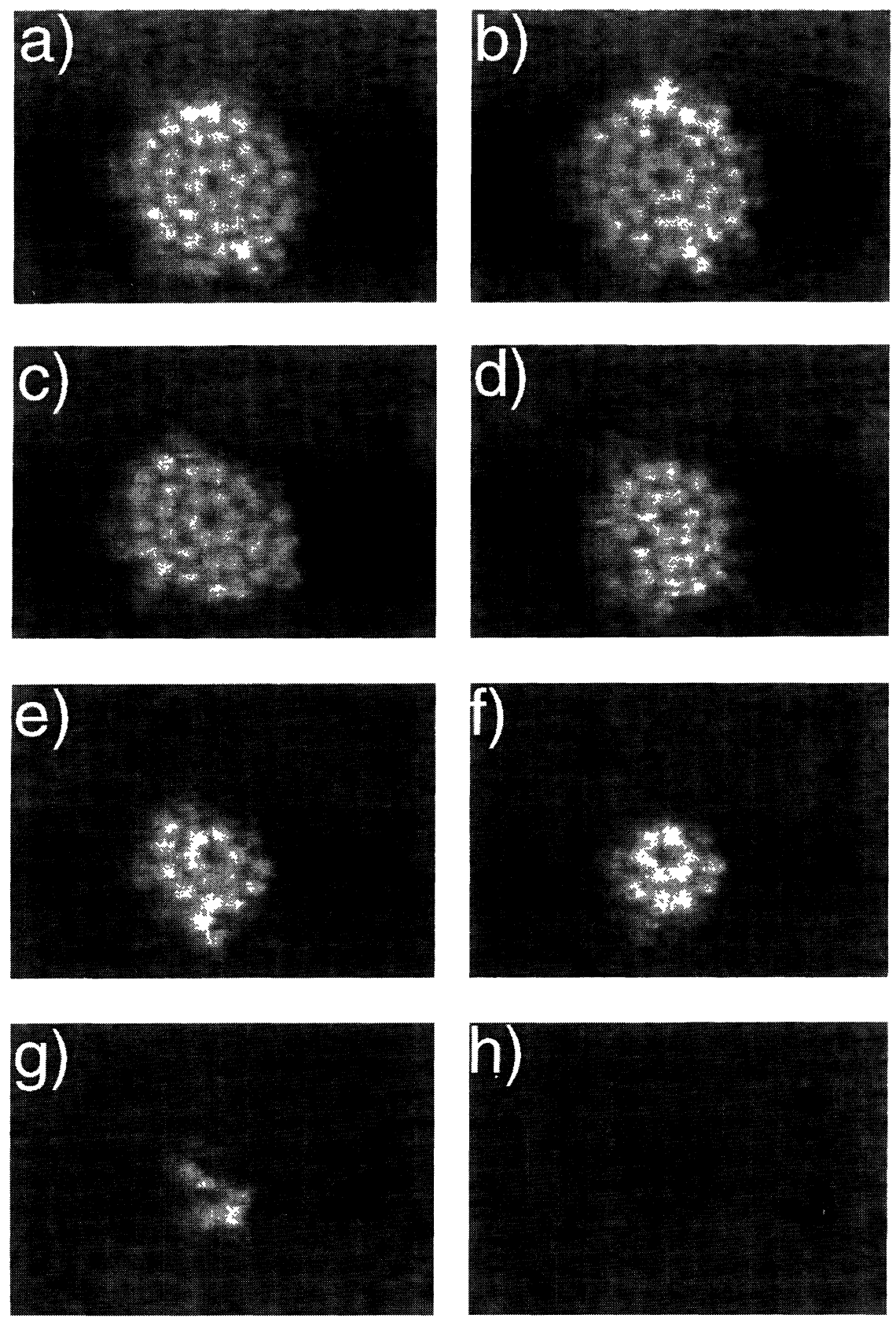

Fig. 7. - Late stage of the decay of an island at $435^{\circ} \mathrm{C}$. Individual adatoms of the reconstruction on the island can be resolved.

within one minute and the surface of the grown film levels out to only four layers. Between figure $4, t=44 \mathrm{~s}$ and figure $4, t=66 \mathrm{~s}$ a small island on top of the right structure dissolves showing directly the flux of atoms across a step edge to lower levels (process I in Fig. 1). The time scale 


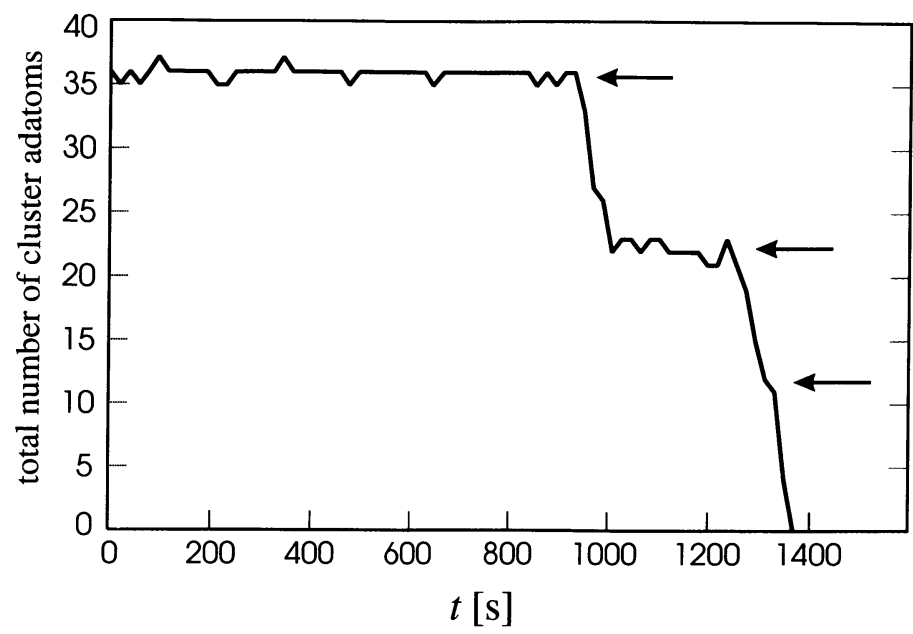

Fig. 8. - Time evolution of the number of atoms in the small Si-cluster shown in figure 7. The arrows point to positions where the island goes to an other metastable state.

on which this leveling out of the surface occurs depends strongly on temperature.

Even a long time after the flux of molecules to the surface has stopped and the surface is in thermodynamic equilibrium, the local structure of the islands is not static. Figure 5 shows the shape fluctuations of an island at $530^{\circ} \mathrm{C}$. The island is nearly in equilibrium with its neighborhood, therefore the overall size of the island changes very little. The shape has changed from a triangle during growth (see Fig. 2) to a hexagon showing that the triangular shape is just caused by the non-equilibrium situation during growth. Although on avearge the shape of the island in figure 5 is hexagonal, the relative fraction of the individual step edge changes on a time scale of minutes. These changes are caused by detachment from and attachment to the step edge (process $\mathrm{H}$ and $\mathrm{C}$ in Fig. 1) and by diffusion of atoms along the step edge (process D in Fig. 1). Close inspection of figure 5 shows that the changes in the shape of the island visible on our time scale of seconds do not occur atom by atom but in portions of the reconstruction unit cell on the island. That means that there are different time scales of atom movements involved, a slow one for complete unit cells of the surface reconstruction and a fast one for the motion of individual atoms along the step edge which is too fast to be observed by STM.

During growth the continuous deposition of material drives the system to a non-equilibrium situation. When this deposition is stopped the system proceeds to a new equilibrium state on a time scale dependent on temperature. Figure 6 shows the state of a surface immediately before (Fig. 6a) and $1400 \mathrm{~s}$ after (Fig. 6b) the deposition has stopped. All islands in a) marked by an arrow have disappeared in b) (detachment of atoms, process $\mathrm{H}$ in Fig. 1). The material which was contained in these islands has contributed to the remaining islands and to the step edge visible in the image. Additionally holes in the lower level (marker A) have been filled. This decay of smaller islands, growth of bigger islands and filling of holes (island coarsening) leads to an overall smoothing of the interface. The physical reason for this behavior is the difference in the detachment rate of atoms from a small island with a large edge to volume ratio compared to a larger island with a smaller edge to volume ratio. For very long times also the bigger islands will decay and all material will contribute to step edges.

The very last stage of the decay of an island is shown in figure 7. Compared to figure 6 here the process is slowed down due to the low temperature of $435^{\circ} \mathrm{C}$. Individual adatoms of this island 
can by resolved and the decay process can be analyzed quantitatively. Figure 8 shows a plot of the adatoms of the island in figure 7 versus times. The atoms do not detach continuously but there exist metastable arrangements which can be connected with the existence of integral numbers of units of the surface reconstruction $((5 \times 5),(7 \times 7),(3 \times 3))$ on the island. The slopes of the periodes of fast transition between two metastable states (marked by arrows in Fig. 8) can be used to estimate the activation energies involved in this detachment process.

In conclusion it is shown that most of the basic steps in epitaxial growth sketched in figure 1 can directly or at least indirectly be observed with time resolved STM. The main problem at the moment is the limited scanning speed of the STM which limits the image acquisition to a few frames per second. Whereas this speed is sufficient to see changes in the island structure at relevant growth temperatures, it is in most cases not sufficient to see the motion of individual atoms at a temperature where ordered epitaxial growth occurs.

\section{References}

[1] Schröder J., Günther C., Hwang R.Q. and Behm R.J., Ultramicroscopy $42-44$ (1991) 475.

[2] Köhler U., Demuth J.E., Hamers R.J., J. Vac. Sci. Technol. A7 (1989) 2860.

[3] Voigtländer B. and Zinner A., Surf. Sci. Lett. 292 (1993) L775.

[4] Tokumoto H., Miki K., Morita Y., Sato T., Iwatsuki M., Suzuki M. and Fukuda F., Ultramicroscopy $42-44$ (1992) 816.

[5] Besenbacher F., Jensen F., Lægsgaard E., Mortensen K. and Stensgaard I., J. Vac. Sci. Technol. 9 (1991) 874.

[6] Andersohn L. and Köhler U., Surf. Sci. 284 (1993) 77.

[7] Venables J.A., Spiller G.D.T. and Hanbücken M., Rep. Prog. Phys. 47 (1984) 399.

[8] Köhler U., Andersohn L. and Dahlheimer B., Appl. Phys. A57 (1993) 491. 